\title{
Lesions of Orbitofrontal Cortex Impair Rats' Differential Outcome Expectancy Learning But Not Conditioned Stimulus-Potentiated Feeding
}

\author{
Michael A. McDannald, ${ }^{\star}$ Michael P. Saddoris, ${ }^{\star}$ Michela Gallagher, and Peter C. Holland \\ Department of Psychological and Brain Sciences, Johns Hopkins University, Baltimore, Maryland 21218
}

\begin{abstract}
Patients with damage to the orbitofrontal cortex (OFC) display various impairments in cognitive and affective function, including a reduced ability to use information about the consequences of their actions to guide their behavior. In this study, rats with neurotoxic lesions of the OFC failed to use specific expectancies about outcomes to guide their learning of an instrumental discrimination task. In contrast, lesioned rats were unimpaired in a measure of learned motivational function, the potentiation of feeding under conditions of food satiation, by a conditioned stimulus that had been paired with food while the rats were food deprived. Notably, performance of both of these tasks has been shown to depend on the function of the basolateral amygdala (BLA), a region that is richly interconnected with the OFC. Thus, the present results are consistent with the view that the acquisition and use of specific outcome expectancies to guide behavior critically involve a neural system that includes the BLA and the OFC, but they indicate that certain motivational properties acquired by cues on the basis of appetitive learning involve BLA circuitry apart from the OFC.
\end{abstract}

Key words: orbitofrontal cortex; outcome expectancy; potentiated feeding; associative learning; motivation; basolateral amygdala

\section{Introduction}

Associative learning has consequences beyond the conditioning of overt motor behavior. Pairing an initially neutral conditioned stimulus (CS) with a reinforcer allows that CS to activate a memorial representation of that reinforcer ("outcome expectancy"), which can have many behavioral effects (Rescorla, 1988). Likewise, associative learning endows a CS with motivational properties. For example, a CS paired with food delivery often acquires the ability to serve as a conditioned reinforcer for the establishment of new learning (Rescorla, 1980) and to modulate the performance of consummatory (Weingarten, 1983) or instrumental (Dickinson et al., 2000) behavior.

Recent studies in rats and monkeys suggest that the orbitofrontal cortex (OFC) is critical to the use of learned outcome expectancies to guide behavior (Pickens et al., 2003; Izquierdo et al., 2004) and to some aspects of learned motivational function (Pears et al., 2003). A common assessment of expectancy is the reinforcer devaluation procedure. In one version of this task, a rat is given light-food pairings, until the light consistently controls behavior directed to a food cup. Next, an aversion is established to the food by pairing it with an illness-inducing agent in the absence of the light. Finally, responding to the light is tested in the absence of the food. Although normal rats show a spontaneous

\footnotetext{
Received Dec. 29, 2004; revised March 16, 2005; accepted March 28, 2005.

This work was supported by National Institutes of Health Grant MH53667. We thank Erin Kerfoot and Vanessa McKenna for technical support.

*M.A.M. and M.P.S. contributed equally to this work.

Correspondence should be addressed to Peter Holland, Department of Psychological and Brain Sciences, Johns Hopkins University, 3400 North Charles Street, Baltimore, MD 21218. E-mail: pch@jhu.edu.

D01:10.1523/JNEUROSCI.5301-04.2005

Copyright $\odot 2005$ Society for Neuroscience $\quad$ 0270-6474/05/254626-07\$15.00/0
}

reduction in food-cup behavior, rats with OFC lesions fail to show such a reduction (Gallagher et al., 1999; Pickens et al., 2003, 2005). This failure is believed to reflect an inability of OFClesioned animals to use a CS-activated expectancy of the newly devalued reinforcer to guide responding.

At the same time, Pears et al. (2003) reported that OFC lesions interfered with learned motivational processes in marmosets. Although lesioned animals showed normal acquisition of instrumental responding reinforced by food, they showed significant deficits in acquiring responding that was reinforced by a previously trained CS. The failure of a CS to serve as a conditioned reinforcer for instrumental responding in OFC-lesioned animals suggested that the OFC plays a critical role in the assignment of motivational value to the CS.

Similar impairments in conditioned reinforcement and in tests for the use of outcome expectancies, such as reinforcer devaluation, have also been seen after lesions of the basolateral amygdala (BLA) (Burns et al., 1993; Hatfield et al., 1996). Because these two regions share rich reciprocal connections (Ghashghaie and Barbas, 2002), the OFC and the BLA form a circuit that may mediate both learned motivational functions and the use of outcome expectancies to guide behavior.

The experiments reported here sought to extend such findings by evaluating the effects of OFC lesions on the performance of rats in two additional associative learning tasks: CS-potentiated feeding (Weingarten, 1983), which is believed to engage learned motivational processes (experiment 1 ), and a differential outcome expectancy (DOE) task (Trapold and Overmier, 1972), in which instrumental performance is mediated by learned expectancies (experiment 2). These tasks were selected on the basis of 
previous research showing them to be sensitive to disruptions in BLA function (Blundell et al., 2001; Holland et al., 2002).

\section{Materials and Methods \\ Subjects}

The subjects were 26 male Long-Evans rats (Charles River Laboratories, Raleigh, NC) that weighed 300-325 g when they arrived at the laboratory vivarium. After 1 week with ad libitum access to food and water in individual cages, the rats were reduced to $85 \%$ of their ad libitum weights by restricting their access to food. The rats lived in individual cages, with ad libitum access to water, in a colony room illuminated from 6:00 A.M. to 8:00 P.M. Experiment 1 was conducted in two replications, one with 18 rats and one with 8 rats, with lesion and behavioral training procedures balanced across replications.

\section{Surgical procedures}

Aseptic surgeries to make bilateral neurotoxic lesions of the OFC were performed under isoflurane (Isovet; Mallinckrodt, Mundelein, IL) anesthesia. The lesions were made using NMDA at a concentration of 20 $\mathrm{mg} / \mathrm{ml}$ in PBS (Sigma, St. Louis, MO) and injected using a glass micropipette attached by a length of plastic tubing to a picospritzer (General Valve Corporation, Fairfield, NJ). OFC lesions were made in 16 rats, using four injection sites in each hemisphere: two injections at $4.0 \mathrm{~mm}$ anterior to bregma, $4.2 \mathrm{~mm}$ ventral from the skull surface at bregma, and placed $2.2 \mathrm{~mm}(0.08 \mu \mathrm{l})$ and $3.7 \mathrm{~mm}(0.08 \mu \mathrm{l})$ from the midline; and two injections at $3.0 \mathrm{~mm}$ anterior to bregma, $5.2 \mathrm{~mm}$ ventral from the skull surface at bregma, and placed $4.2 \mathrm{~mm}(0.08 \mu \mathrm{l})$ and $3.2 \mathrm{~mm}(0.05 \mu \mathrm{l})$ from midline. For 10 control rats, the glass micropipette was lowered to the same sites, but no injections were given.

\section{Apparatus}

The behavioral training apparatus for experiment 1 consisted of four individual chambers $(22.9 \times 20.3 \times 20.3 \mathrm{~cm})$ with aluminum front and back walls, clear acrylic sides and top, and a floor made of $0.48 \mathrm{~cm}$ stainless-steel rods spaced $1.9 \mathrm{~cm}$ apart. A dimly illuminated food cup was recessed in the center of one end wall. An infrared photocell placed just inside the food cup was polled $(1 \mathrm{kHz})$ by computer circuitry. Each chamber was enclosed in a sound-resistant shell. A speaker, used to present an auditory CS, was mounted on the inside wall of the inner shell, $10 \mathrm{~cm}$ above the experimental chamber and even with the end wall opposite the food cup. Ventilation fans provided masking noise (70 dB). Constant dim illumination was provided by a $6 \mathrm{~W}$ lamp behind a dense red lens mounted next to the speaker. A television camera was mounted within each shell to provide a view of the chamber. Television images were recorded in selected sessions, but the analysis of these recordings is not presented. The apparatus used in experiment 2 comprised eight chambers similar to those used in experiment 1 . These chambers included three visual CS sources: a jeweled $6 \mathrm{~W}$ lamp located $9.5 \mathrm{~cm}$ above the food cup and two $6 \mathrm{~W}$ lamps located on opposite side walls of the sound-attenuating shell, each centered $10 \mathrm{~cm}$ above the experimental chamber. In addition, any of three operant response devices could be inserted into each chamber. A $2 \times 2 \mathrm{~cm}$ lever could be positioned $5 \mathrm{~cm}$ above the floor, $2.5 \mathrm{~cm}$ to the left of the food cup; a $10 \mathrm{~cm}$ chain could be suspended from the ceiling to within $10 \mathrm{~cm}$ of the floor, $3.5 \mathrm{~cm}$ to the left of the food cup; and rats could be given access to a $2.5 \mathrm{~cm}$ port with a paddle behind it, $2.5 \mathrm{~cm}$ to the right and $9 \mathrm{~cm}$ above the food cup.

\section{Behavioral training procedures}

Experiment 1 examined the performance of sham-lesioned and OFClesioned rats in a potentiated feeding procedure, which assessed the ability of a CS to promote feeding while the rats were food sated. Hungry rats were first trained with a pavlovian discrimination procedure, in which one auditory CS was paired with food and another was nonreinforced. After an extended period of food satiation, food consumption in the presence of the previously reinforced CS and the nonreinforced CS was examined. In previous studies, normal rats ate more during the reinforced CS than during the nonreinforced CS or during baseline periods (Holland et al., 2002; Petrovich et al., 2002; Holland and Gallagher, 2003), but rats with lesions of the BLA did not show this potentiation of feeding. Those studies also showed that the enhanced consumption dur- ing a reinforced CS was independent of food-cup approach responses conditioned to the CS: feeding was potentiated regardless of where food was placed in the chamber, and brain lesions that eliminated potentiated feeding had no effect on the approach to the food cup, even in tests in which food was present in that cup.

All training sessions lasted $32 \mathrm{~min}$ and began daily at approximately the same time (9:00 A.M.). During the first two sessions, the rats were trained to eat from the recessed food cup. In each of these sessions, there were eight deliveries of the reinforcer used throughout experiment 1: two $45 \mathrm{mg}$ food pellets (formula A; Research Diets, New Brunswick, NJ) given $0.5 \mathrm{~s}$ apart. In these and all remaining training sessions, the intertrial intervals were variable (mean, $4 \mathrm{~min}$ ) within a range of $2-6 \mathrm{~min}$. The rats then received training sessions to establish a discrimination between two auditory CSs. In each session of the first part of this training, one-half of the rats received eight reinforced $10 \mathrm{~s}$ presentations of an $80 \mathrm{~dB}$ white noise; the other half received eight reinforced presentations of a $10 \mathrm{~s}, 80$ $\mathrm{dB}, 1500 \mathrm{~Hz}$ tone. In the second portion of this training, the rats received two reinforced presentations of the CS that they had received in the first part of training $(\mathrm{CS}+)$, randomly intermixed with six nonreinforced presentations of the other auditory stimulus (CS-). Because of experimenter error, the rats in the first replication received two initial CS+ training sessions and 12 discrimination training sessions, and the rats in the second replication received four initial CS + training sessions and eight discrimination training sessions.

The rats were then given ad libitum access to food in their home cages for $9 \mathrm{~d}$. On each of the last $2 \mathrm{~d}$, the rats received a consumption test in the experimental chambers. Each consumption test began with a $10 \mathrm{~min}$ pretest of pellet consumption in the chambers before the presentation of a CS. In previous studies of potentiated feeding, we found that when satiated rats were placed in the experimental chambers, they often ran immediately to the food cup and ate any food there. The pretest was intended to reduce the contribution of this effect to feeding during the CS presentations. In the pretest, 50 pellets were present in the recessed food cup when the rats were placed in the chambers. After $10 \mathrm{~min}$ had elapsed, the rats were removed from the chambers and placed in transport cages. Food pellets were suctioned from the food cup and trays beneath the chamber floor and saved for counting. The food cups were then quickly refilled with 50 new pellets, and the rats were returned to the chambers for a 10 min test of consumption in the presence of the CS + or CS - . During that test, there were $10 \mathrm{CS}+$ or CS - presentations, each of $10 \mathrm{~s}$ duration. No additional pellets were delivered after CS+. Both the reinforcement contingency in training ( $\mathrm{CS}+$ or $\mathrm{CS}-$ ) and the identity of the CS (tone or noise) were counterbalanced over the two test sessions. Finally, at the end of the $10 \mathrm{~min}$ CS consumption test, the rats were quickly removed, and the remaining pellets were suctioned and reserved for counting. Behavior was recorded by a video camera throughout each test, but analyses of the behavior are not presented here.

Experiment 2 examined the performance of these same rats in an instrumental discrimination task in which specific outcome expectancies controlled by discriminative stimuli could be used to guide instrumental behavior. Previous studies showed that instrumental discriminations that involve multiple stimuli, responses, and reinforcers (outcomes) are learned more rapidly if each stimulus-response combination is consistently related to a unique outcome (Trapold and Overmier, 1972; Blundell et al., 2001) than if the stimulus-response combinations are randomly reinforced with both outcomes. This observation has been attributed to subjects' use of learned outcome expectancies to supplement the explicit stimuli as cues to guide behavior. Consider a discrimination in which one response (R1) is reinforced only in the presence of one stimulus (S1) and another response (R2) is reinforced only in the presence of a second stimulus (S2). If correct responding during each stimulus is associated, in addition, with a unique outcome, then the unique outcome expectancies that each stimulus elicits can serve as supplemental discriminative cues to signal the correct response; only one response is reinforced in the presence of each expectancy. In contrast, if correct responding during each stimulus is randomly associated with both outcomes, then each response is reinforced in the presence of both expectancies, detracting from the ability of the explicit cues ( $\mathrm{S} 1$ and S2) to control correct responding via differential outcome expectancies. 
In experiment 2, each rat was given training that involved two different reinforcement outcomes (flavored sucrose solutions), three different visual CSs, and three different operant responses. As indicated in Table 1, two of the stimulus-response combinations consistently led to unique outcomes (S1-R1-O1 and S2-R2-O2), whereas the third stimulus-response combination was reinforced with each outcome $50 \%$ of the time (S3-R3-O1/O2).

The identities of $\mathrm{O} 1$ and $\mathrm{O} 2(0.3 \mathrm{ml}$ of a solution of $1 \mathrm{~g}$ of either orange or grape unsweetened Kool-Aid (Kraft Foods, Northfield, IL) and $50 \mathrm{~g}$ of sucrose in $1 \mathrm{~L}$ of water), S2 and S3 (illumination of the jeweled lamp, flashing at a rate of $3 \mathrm{~Hz}$, or the steady illumination of the lamp on the left side of the chamber), and R2 and R3 (lever press or chain pull) were completely counterbalanced. S1 was the illumination of the lamp on the right side of the chamber, and R1 was paddle pushing for all rats. The performance of an inappropriate response (e.g., R1 at any time other than during S1) had no consequence.

Rats were first shaped to perform each of the three operant responses needed for differential outcome expectancy training. In each shaping session, only one of the operant responses was available. To encourage contact with the response device in these initial training sessions, it was covered with a paste of sucrose, food-pellet dust, and water. In the first session, performance of each R2 was reinforced with $\mathrm{O} 2$. The session ended for each rat after it reached 50 responses. In the second session, each R3 was reinforced with O1, until 25 responses were emitted; in the third session, each R3 was reinforced with $\mathrm{O} 2$ until 25 responses were emitted; and in the fourth session, each R1 was reinforced with $\mathrm{O} 1$ until 50 responses were emitted.

Next, rats were trained to emit each of these responses only in the presence of a particular visual signal over the course of nine $32 \mathrm{~min}$ sessions. In each of these sessions, only one response device was available. In the first three sessions, R1 was reinforced (with O1) during $1630 \mathrm{~s}$ presentations of $\mathrm{S} 1$; in the next three sessions, R2 was reinforced (with $\mathrm{O} 2$ ) during $1630 \mathrm{~s}$ presentations of S2; and in the final three sessions, R3 was reinforced (50\% with $\mathrm{O} 1$ and $50 \%$ with $\mathrm{O} 2$ ) during the 30 s presentation of S3. Any rats that failed to perform a response were given an extra operant response training session without signals on that response before the next training session.

In the DOE test phase (shown in Table 1), the rats received five $30 \mathrm{~min}$ sessions of discrimination training, in which all three responses devices were available, and all three $30 \mathrm{~s}$ signals were presented in each session. In each of these sessions, the rats received eight S1-R1-O1 trials, in which each R1 during S1 was reinforced with O1, eight S2-R2-O2 trials, in which each R2 during S2 was reinforced with O2, and eight S3-R3$\mathrm{O} 1 / \mathrm{O} 2$ trials, in which each $\mathrm{R} 3$ during $\mathrm{S} 3$ was reinforced with either $\mathrm{O} 1$ (four trials) or O2 (four trials). The performance of any response other than the correct, reinforced response was recorded as an error but had no consequence.

To provide evidence of the ability of the lesioned rats to discriminate between the two reinforcers, we administered a test of discriminative taste-aversion learning to all of the rats at the conclusion of the experiment. On each of two training days, separated by $1 \mathrm{~d}$ during which there was no training, each rat received $5 \mathrm{~min}$ access to $12 \mathrm{ml}$ of either orangeor grape-flavored sucrose in the liquid cups, paired with a $5 \mathrm{ml} / \mathrm{kg}$ injection of $0.3 \mathrm{~m}$ lithium chloride. Discriminative taste-aversion learning was assessed by measuring the rats' consumption of each of the two flavored sucrose solutions. On the second and third days after the final flavortoxin pairing, the rats received 5 min consumption tests in which $12 \mathrm{ml}$ of solution was made available in the liquid cups. The order of solution presentation was counterbalanced.

\section{Response measures}

Experiment 1. The measure of conditioning to an auditory CS during the pavlovian training phase was the percentage of time during which the food cup photobeam was broken (presumably indicating that a rat's head was in the food cup) during the last $5 \mathrm{~s}$ of each CS interval. In addition, the percentage of time in the food cup during the $5 \mathrm{~s}$ intervals immediately before each trial was recorded as a measure of baseline responding. The measure of consumption was the number of food pellets eaten, which was measured by subtracting the number of whole pellets remain- ing in the food cups and trays from 50. In the rare case of a rat's leaving fractional pellets uneaten, a fractional pellet was arbitrarily defined as a 0.5 pellet. In no case did a rat leave more than two fractional pellets. We reported the number of pellets consumed in the pretest periods and during the separate CS+ and CS - tests. The difference between the number of pellets consumed in the CS + and CS - tests served as a measure of the effect of the past conditioning relationship of the CS with food on consumption in each rat and thus served as a direct, within-subject measure of the potentiated feeding effect.

Experiment 2. For each rat, we recorded the percentages of each trial type (S1, S2, or S3) during which each response (R1, R2, or R3) occurred as well as the percentage of dummy trials (empty intervals equal in duration to the trials, immediately before each trial) during which each of those responses occurred. The primary measure of discriminative responding in the DOE test phase was responding during S1. The correct (reinforced) response during S1 was R1; the critical data were the likelihood of R2 and R3 errors during S1. If learned expectancies of $\mathrm{O} 1$ and $\mathrm{O} 2$ were used as discriminative cues to direct responding, then R3 errors would be more frequent than R2 errors, because R1 and R3 shared the outcome O1, whereas R1 and R2 produced different outcomes. Thus, for responding during S1, R2 was defined as the "different-outcome error" (see Table 1, bold type), and R3 was termed the "shared-outcome error." Comparable measures were constructed for responding during S2, in which R2 was the correct response, R1 was the different-outcome error (see Table 1, bold type), and R3 was the shared-outcome error; however, we used responding during S1 as our primary measure, because S1 was the same stimulus for all rats, and S2, S3, R2, and R3 were perfectly counterbalanced among all rats.

\section{Histological procedures}

After completion of the behavioral testing in experiment 2, the rats were deeply anesthetized with Nembutal $(150 \mathrm{mg} / \mathrm{kg})$ and perfused with $0.1 \mathrm{M}$ PBS, followed by $10 \%(\mathrm{v} / \mathrm{v})$ Formalin. The brains were removed and stored in $0.1 \mathrm{M}$ PBS with $20 \%(\mathrm{w} / \mathrm{v})$ sucrose at $4^{\circ} \mathrm{C}$ for $24-48 \mathrm{~h}$. Sections $(40 \mu \mathrm{m})$ were taken from each brain, and alternate sections were mounted on slides and Nissl stained to verify the lesions.

\section{Results}

\section{Histological results}

Ten rats had acceptable lesions of the OFC encompassing the lateral and dorsolateral orbital regions, the lateral portions of the ventral orbital cortex, and portions of both the dorsal and ventral agranular insular cortex. This target region was designed to include areas on the dorsal bank of the rhinal sulcus that have reciprocal interactions with the BLA (Krettek and Price, 1977; Kita and Kitae, 1990; Shi and Cassell, 1998) but to exclude the medial orbital and medial portions of the ventral orbital cortex and gustatory regions located in the agranular insular cortex posterior to the genu of the corpus callosum (Saper, 1982; Kosar et al., 1986a,b; Krushel and Van der Kooy, 1988). The lesions averaged $60 \%$ of the damage of the target region bilaterally. In most brains, damage was substantial along the anteroposterior axis of the target region from 2.7 to $4.7 \mathrm{~mm}$ anterior to bregma. No lesion extended anterior to the coronal plane +5.0 or posterior to the plane +2.2 relative to bregma. Photomicrographs of a representative lesion and sham brain are shown in Figure 1. Six lesioned rats showed little evidence of damage and were discarded from the analyses. There was little discernable damage in any of the 10 sham-lesioned control rats.

\section{Behavioral results}

\section{Experiment 1: pavlovian discrimination training}

Two lesioned rats and one sham rat failed to acquire the auditory discrimination, and their data were discarded from experiment 1 , leaving eight OFC-lesioned and nine sham rats. The OFC lesion did not affect auditory pavlovian discrimination learning, which 


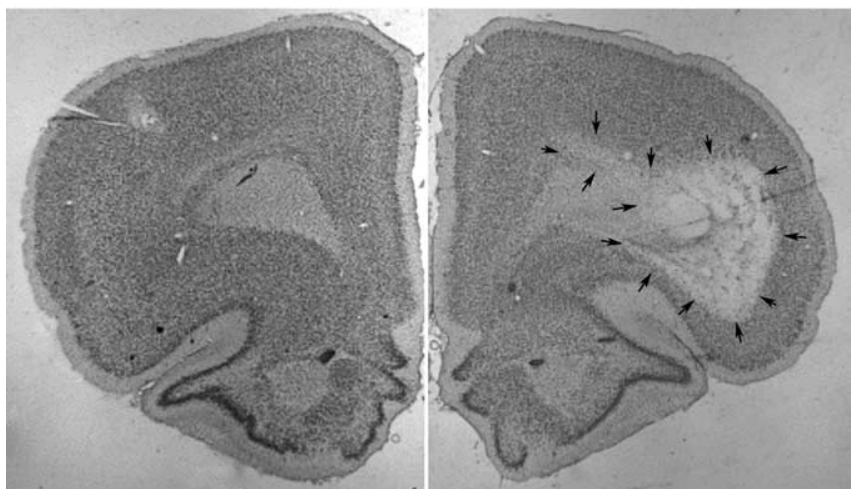

Figure 1. Photomicrographs showing typical sham (left hemisphere) and neurotoxic (right hemisphere) lesions of the orbitofrontal cortex. Arrows outline the lesion.

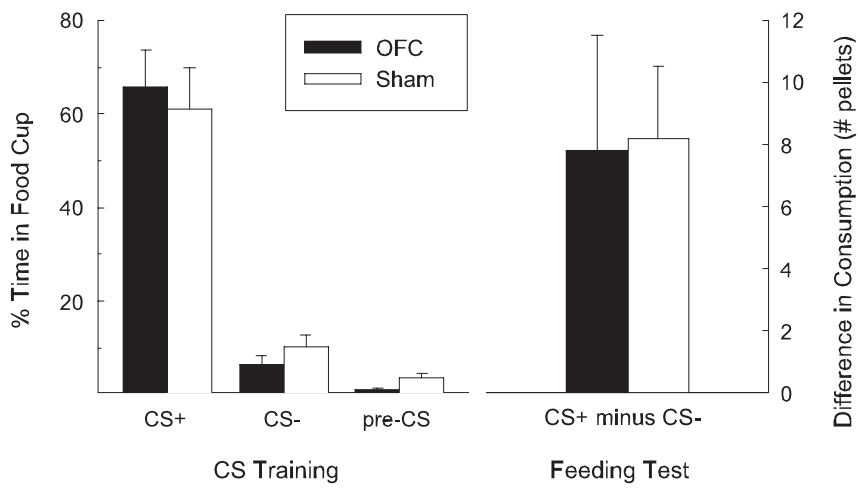

Figure 2. Left, Mean \pm SEM food cup conditioned responding during the reinforced ( $C S+$ ) and nonreinforced (CS - ) conditioned stimuli over the last four sessions of experiment 1. Right, Potentiated feeding consumption test results of experiment 1. Error bars indicate the mean \pm SEM difference between pellet consumption during the test session that included the previously reinforced CS and consumption during the test with the nonreinforced CS.

was acquired rapidly in all remaining rats. Figure 2 (left) shows performance in the final four training sessions. A lesion (OFC or sham) by stimulus (reinforced or nonreinforced CS) ANOVA showed a significant effect of stimulus $\left(F_{(1,15)}=113.96\right)$ but no effect of lesion or lesion by stimulus interaction $(F<1)$.

\section{Experiment 1: consumption tests}

The primary data of this experiment are the results of the consumption tests with CS + and CS - . Figure 2 (right) shows consumption difference scores (CS+ minus CS - ) for OFC-lesioned and sham-lesioned rats. Both sham and lesioned rats ate more pellets in the test in which the previously reinforced CS+ was presented (15.6 \pm 5.9 and $19.4 \pm 4.6$ pellets, respectively) than in the test with the nonreinforced CS $-(7.4 \pm 4.3$ and $11.6 \pm 5.9$ pellets $)$. Thus, the ability of the noise and tone stimuli to potentiate eating depended on their associative history, that is, their previous pairing with food. A lesion by test (CS+ or CS - ) ANOVA showed only a main effect of test $(\mathrm{CS}+\mathrm{vs} \mathrm{CS}-): F_{(1,15)}=15.85 ; p=0.001$; remaining $F<1$. Likewise, a contrast of the difference scores portrayed in Figure $2 B$ showed no effect of lesion; $F<1$.

Pretest food consumption also did not differ as a function of the lesion, averaging $29.6 \pm 4.2$ pellets in OFC-lesioned rats and $30.4 \pm 3.6$ pellets in sham-lesioned rats; $F<1$. Finally, the lesions had no effect on the rats' test weights or weight gain during the satiation phase; $F<1$.

\section{Experiment 2}

Three OFC-lesioned and two sham rats failed to acquire one or more of the three operants in the initial response-shaping ses-
Table 1. Differential outcome expectancy test procedures of experiment 2

\begin{tabular}{lll}
\hline Stimulus & Correct response $\rightarrow$ outcome & Errors \\
\hline S1 & $\mathrm{R} 1 \rightarrow 01$ & $\mathbf{R 2}-, \mathrm{R} 3-$ \\
S2 & $\mathrm{R} 2 \rightarrow 02$ & $\mathbf{R} 1-, \mathrm{R} 3-$ \\
S3 & $\mathrm{R} 3 \rightarrow 01 / 02$ & $\mathrm{R} 1-, \mathrm{R} 2-$
\end{tabular}

In each of the five sessions of discrimination training, three discriminative stimuli were presented, and three response devices were available. 01 and 02 were two flavored sucrose solutions used as reinforcing outcomes (counterbalanced), R1 was a paddle-push response, and R2 and R3 were lever-press and chain-pull responses (counterbalanced). Bold type indicates the different-outcome error (see Results). S1 was the illumination of a visual stimulus on one side of the chamber, and S2 and S3 were two other visual stimuli (counterbalanced). $\rightarrow$ indicates a response-outcome contingency; - indicates no scheduled consequence of a response.
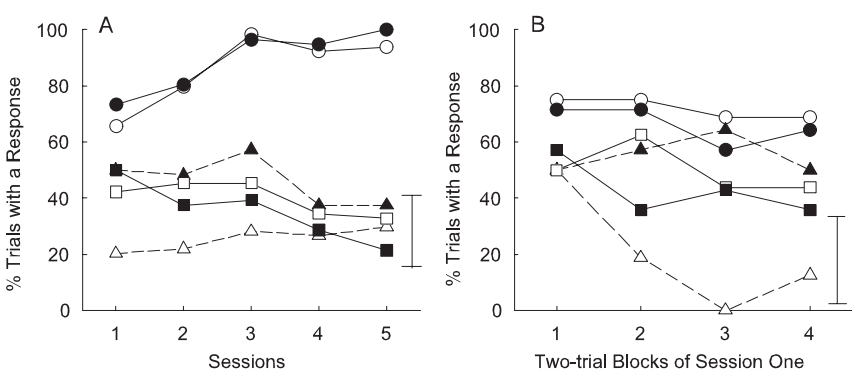

$$
\begin{aligned}
& -\bigcirc \text { Sham R1 Correct } \\
& -\triangle \text { OFC R1 Correct } \\
& \text { Sham R2 Different error } \\
& \square \text { OFC R2 Different error } \\
& \square \text { Sham R3 Shared error } \\
& \text { OFC R3 shared error }
\end{aligned}
$$

Figure 3. Acquisition of instrumental responding during stimulus $\mathrm{S} 1$ in the DOE test phase of experiment 2. $\boldsymbol{A}$, Mean responding in each session. $\boldsymbol{B}$, Acquisition of responding within the first test session alone. The error bars provide measures of variance appropriate to the error terms for the lesion by error type interactions: 2 (mean square error/df) ${ }^{1 / 2}$.

sions; they were eliminated from experiment 2, leaving seven OFC-lesioned and eight sham rats. The lesions did not affect acquisition of responding under the control of the three visual signals during the initial conditioning sessions, in which only one signal and its correct response device were available. By the last of each of these sessions, the percentage of trials on which at least one response occurred was $84.4 \pm 6.6,88.3 \pm 7.1$, and $90.6 \pm$ $6.0 \%$ during S1, S2, and S3, respectively, in the lesioned rats, and $86.6 \pm 8.9,89.3 \pm 5.9$, and $88.4 \pm 6.6 \%$ in the sham rats.

The primary data of experiment 2 were the acquisition of the test discriminations under the conditions shown in Table 1. Figure 3 shows performance during S1, in which R1 responses were always reinforced with $\mathrm{O} 1$, and errors, consisting of R2 or R3 responses, were not reinforced. R2 responses were always reinforced with a different outcome (O2) on S2 trials, whereas R3 was reinforced with the same outcome $(\mathrm{O} 1)$ as $\mathrm{R} 1$ on one-half of the $\mathrm{S} 3$ presentations and with $\mathrm{O} 2$ on the other half of the $\mathrm{S} 3$ trials. Over all five sessions (Fig. 4A), sham rats showed more sharedoutcome errors (R3) than different-outcome errors (R2) (Table 1 , bold type) during S1. This result can be attributed to the rats' use of specific outcome expectancies to guide responding: responses reinforced in the presence of different expectancies (e.g., $\mathrm{R} 1$ and R2) were differentiated more readily than responses that yielded the same outcome (e.g., R1 and R3). In contrast, OFClesioned rats showed equivalent levels of shared- and differentoutcome errors. The absence of any difference in shared- and different-outcome errors in these rats is consistent with an impairment in the associative basis for outcome expectancy learning.

The levels of correct $\mathrm{R} 2$ responses on S2 trials (86.6 $\pm 10.9 \%$ in sham rats and $79.6 \pm 15.8 \%$ in lesioned rats) and $\mathrm{R} 3$ responses 

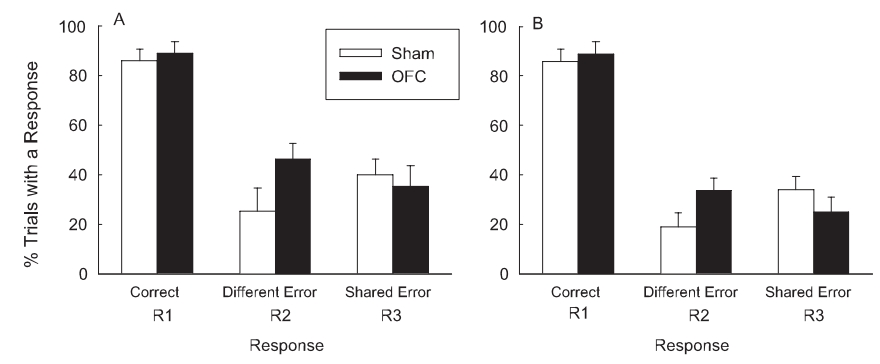

Figure 4. Overall mean \pm SEM responding during stimulus $S 1$ in the DOE test phase of experiment 2. A, Percentage of trials on which each type of response occurred at any time during S1. $\boldsymbol{B}$, Only responding that occurred before the delivery of the first reinforcer on each trial is shown.

on S3 trials $(88.1 \pm 10.0$ and $85 \pm 16.3 \%$, respectively) did not differ among either responses or groups. Finally, Figure $3 B$, which portrays successive two-trial blocks of responding during the first session, shows that the differences in responding during the first training session reflected rapid acquisition of the differences in error frequencies rather than different initial levels of those responses.

It might be argued that the sham rats were using cues provided by the reinforcing outcomes themselves to guide instrumental responding rather than S1-evoked expectancies of those outcomes (Blundell et al., 2001). In that case, the deficit in the performance of the lesioned rats would reflect a deficit in their ability to use overt flavor cues, rather than flavor expectancies, to guide responding. To evaluate that possibility, we reanalyzed the data shown in Figure 4A, excluding responses that occurred after the delivery of the first reinforcer on each trial. The remaining responses could not have been cued by the physical reinforcer delivered on that trial and thus reflect more clearly the influence of S1-evoked outcome expectancies. These data (Fig. 4B) showed a pattern similar to those from the entire stimulus duration (Fig. 4A).

A lesion by error (R2 or R3) by sessions ANOVA of the data shown in Figure $3 A$ (and summarized in Fig. $4 A$ ) showed a significant lesion by error interaction: $F_{(1,13)}=6.47 ; p=0.024$. Individual post hoc contrasts [Tukey's honestly significant difference (HSD) procedure; $p<0.05$ ] revealed that the sham rats showed significantly fewer R2 different-outcome errors than R3 shared-outcome errors and also significantly fewer R2 differentoutcome errors than the OFC-lesioned rats. No other contrast was significant. A comparable ANOVA of the data that excluded postreinforcement responses (Fig. $4 B$ ) also showed a reliable lesion by error interaction: $F_{(1,13)}=11.83 ; p=0.004$. As with the analysis of the overall data, individual Tukey's HSD procedure $(p<0.05)$ contrasts revealed that the sham rats showed significantly fewer R2 different-outcome errors than R3 sharedoutcome errors and also significantly fewer R2 different-outcome errors than the OFC-lesioned rats. No other contrast was significant, although the numerically lower levels of R3 errors in OFClesioned rats than in sham-lesioned rats approached significance: $p=0.082$.

Comparable analyses of performance during S2 (data not shown in Figs. 3 or 4) were also performed. For those analyses, R2 was the correct response, R1 was the different-outcome error, and R3 was the shared-outcome error. As with the analyses of S1 responding, sham-lesioned rats showed more shared-outcome (R3) errors $(49 \pm 4.1 \%)$ than different-outcome (R1) errors $(29.7 \pm 8.5 \%)$, but OFC-lesioned rats did not $(39.3 \pm 6.9$ and
$39.9 \pm 8.1 \%)$. Statistical analyses like those performed on S1 responding yielded comparable outcomes.

Finally, although the lesioned rats showed no evidence of using differential outcome expectancies to guide their instrumental responding, they learned the final flavor aversion discrimination as well as the shams. Thus, their deficiency in using outcome expectancies is not attributable to an inability to discriminate between the two outcomes. The OFC-lesioned rats consumed means of $2.7 \pm 0.5 \mathrm{ml}$ of the devalued flavor and $6.8 \pm 1.5 \mathrm{ml}$ of the nondevalued flavor, and the sham-lesioned rats consumed $2.7 \pm 0.7$ and $7.2 \pm 0.7 \mathrm{ml}$, respectively. A lesion by flavor ANOVA showed a significant effect of flavor (toxin-paired or not presented in training $)\left(F_{(1,13)}=28.99 ; p<0.001\right)$ but no effect of lesion or its interaction with flavor $(F<1)$.

\section{Discussion}

In this study, rats with neurotoxic or sham lesions of the OFC were tested in both a CS-potentiated feeding task and a DOE task. Although rats with OFC lesions showed normal CS-potentiated feeding in experiment 1 , their performance on the DOE task was substantially impaired in experiment 2 . In that experiment, rats were trained to make different cue-specific instrumental responses, which led to distinct reinforcing outcomes (two flavors of sucrose solution). Although the pattern of errors observed showed that sham-lesioned rats used cue-elicited outcome expectancies to help guide their instrumental responding, OFClesioned rats showed no evidence of using outcome expectancies to guide their behavior. This failure was not attributable to a deficit in discriminating between the two outcomes themselves, because the lesioned rats showed no deficit in discriminating between the two flavored outcomes in a flavor-aversion learning task conducted at the end of the study.

The OFC lesion deficit observed in experiment 2 is consistent with many accounts of OFC function, including those that emphasize the role of the OFC in the representation of information about rewarding outcomes (Rolls, 2000; Montague and Berns, 2002), those that focus on its role in response selection (Arana et al., 2003), and those that point to its involvement in response inhibition (Dias et al., 2000). Expectancy-guided behavior in the DOE task required learned associative access to specific representations of the two reinforcing outcomes by the visual cues, the selection of an instrumental response appropriate to the outcome anticipated on each trial, and the suppression of responses inappropriate to that outcome. Thus, in principle, OFC lesioninduced deficits in any of these processes might underlie the lesion effects observed in experiment 2; however, it is notable that OFC-lesioned rats were no poorer than sham-lesioned rats at suppressing shared-outcome errors. Instead, lesioned rats were only deficient at using outcome expectancies to suppress the performance of a response (the different-outcome error) that was never appropriate to the anticipated outcome. Thus, the deficit observed here implicates the OFC in the acquisition or use of specific outcome expectancies, rather than a more general inhibitory function.

OFC neurons have been demonstrated to selectively code properties of expected rewards, such as their magnitude and valence, in many experimental settings (Schoenbaum et al., 1998, 1999; Tremblay and Schultz, 1999). It is notable that the two outcomes used in experiment 2 differed only in flavor; they had equal caloric value and were equally preferred. Thus, the OFC was important for acquiring or using expectancies that coded sensory properties of the reinforcing outcomes. The OFC is well positioned for such a role, because it is richly interconnected with 
brain regions that process the gustatory and olfactory information necessary to distinguish between the two outcomes used in experiment 2, as well as visual information provided by the discriminative stimuli (Carmichael and Price, 1995a,b).

Holland and Gallagher (2004) noted that the acquisition of outcome expectancy information and the use of that information to guide behavior may depend not only on OFC function but also on the interaction of the OFC with the BLA. For example, performance in the devaluation task (described in Introduction) is disrupted by lesions of either the BLA (Hatfield et al., 1996; Balleine et al., 2003) or the OFC (Gallagher et al., 1999; Pickens et al., 2003). Similarly, the impaired performance of OFC-lesioned rats in the DOE task of experiment 2 complements observations of deficits in DOE performance after BLA lesions. Blundell et al. (2001) found that BLA lesions disrupted performance in a simpler, between-subjects version of the DOE task, and in an unpublished study, we found BLA lesion deficits using procedures identical to those of experiment 2. Finally, Schoenbaum et al. (2003) found that a neural correlate of outcome expectancy that emerges in the OFC in the presence of a predictive cue depends critically on BLA integrity. Such encoding failed to emerge in OFC recordings ipsilateral to a neurotoxic lesion in the BLA when rats used different odor cues to predict the occurrence of either a positive (sucrose) or a negative (quinine) reinforcer. Together, these data provide additional support for the view of Holland and Gallagher (2004).

In contrast to the behavioral deficits produced by OFC lesions in experiment 2, OFC damage in experiment 1 had no effect on the ability of a pavlovian CS, trained with a food reinforcer while the rats were food deprived, to enhance feeding after 1 week of food satiation. Coupled with the results of experiment 2, the failure to find OFC lesion effects in experiment 1 is of interest in constraining the basis for CS-potentiated feeding. Specifically, associatively activated representations of outcome information apparently do not play a critical role in CS-potentiated feeding. For example, it might otherwise be argued that a CS potentiates feeding while sated by activating a memorial representation of the value of the food when the rat was in the deprived state at the time of the original training. Because OFC damage interfered with associatively activated outcome representations in these same rats in experiment 2, it is unlikely that such information was involved in the potentiated feeding observed with the procedures of experiment 1 .

Although we found no effects of OFC lesions on CSpotentiated feeding, others have suggested that the OFC serves an important motivational function in associative learning, whereby previously neutral cues acquire motivational value as a result of pairings with reinforcers (Baxter and Murray, 2002; Pears et al., 2003). Furthermore, these authors suggested that this learned motivational function of the OFC depends on its interaction with the BLA, as we discussed earlier in the case of learned outcome expectancies. Consistent with this claim, although a CS paired with food typically acquires the ability to serve as a conditioned reinforcer (Fantino, 1977; Rescorla, 1980), that ability is impaired in animals with lesions of either the BLA (Cador et al., 1989; Burns et al., 1993; Hatfield et al., 1996; Parkinson et al., 2001) or the OFC (Pears et al., 2003). It seemed reasonable to anticipate that another BLA-dependent motivational function acquired by a CS paired with food, the potentiation of feeding in satiation (Holland et al., 2002), would also critically involve the OFC; however, the results of experiment 1 indicate that BLAOFC connectivity is not critical to this function.

This discrepancy between the effects of OFC lesions on poten- tiated feeding and on conditioned reinforcement has implications for our understanding of both the function of BLA-OFC systems and the nature of these two learning phenomena. Although both of these phenomena are described as reflecting acquired motivational functions of a CS and both are dependent on BLA function, they differ in a number of important ways. For example, potentiated feeding involves enhancement of a speciestypical consummatory response elicited by an innate reinforcer, whereas conditioned reinforcement involves assessment of the ability of a cue to support the acquisition or maintenance of an arbitrary instrumental response. It is possible that for a cue to serve as a conditioned reinforcer, it must maintain access to the value of the original primary reinforcer from which the conditioned reinforcement value was derived, a process known to be OFC dependent (Pickens et al., 2003). At the same time, modulation of feeding itself by learned cues may involve more direct access to neural systems that control ingestive behavior. Thus, the role of the BLA in potentiated feeding may parallel somewhat the role of the amygdala in other learned control of innate behavioral systems, such as conditioned fear. Indeed, Petrovich et al. (2002) found that CS-potentiated feeding, but not conditioned reinforcement, depends on BLA projections to the lateral hypothalamus. Notably, this pattern is the opposite of that found for the OFC (experiment 1) (Pears et al., 2003).

A better understanding of the basis for abnormalities often reported in patients with OFC damage (Bechara et al., 1997; Dolan, 1999) is emerging from the use of behavioral tasks as analytic tools in experimental animals. Such research is shedding light on how information processing within broader neural systems subserves functions that depend on the OFC. The features of such OFC-dependent functions that can be modeled in various tasks will help to provide a theoretical basis for understanding the role of this system in cognition and affect.

\section{References}

Arana FS, Parkinson JA, Hinton E, Holland AJ, Owen AM, Roberts AC (2003) Dissociable contribution of the human amygdala and orbitofrontal cortex to incentive motivation and goal selection. J Neurosci 23:9632-9638.

Balleine BW, Killcross AS, Dickinson A (2003) The effect of lesions of the basolateral amygdala on instrumental conditioning. J Neurosci 23:666-675.

Baxter MG, Murray EA (2002) The amygdala and reward. Nat Rev Neurosci 3:563-573.

Bechara A, Darmasio H, Tranel D, Damasio AR (1997) Deciding advantageously before knowing the advantageous strategy. Science 275:1293-1294.

Blundell P, Hall G, Killcross S (2001) Lesions of the basolateral amygdala disrupt selective aspects of reinforcer representation in rats. J Neurosci 21:9018-9026.

Burns LH, Robbins TW, Everitt BJ (1993) Differential effects of excitotoxic lesions of the basolateral amygdala, ventral subiculum and medial prefrontal cortex on responding with conditioned reinforcement and locomotor activity potentiated by intra-accumbens infusions of D-amphetamine. Behav Brain Res 55:167-183.

Cador M, Robbins TW, Everitt BJ (1989) Involvement of the amygdala in stimulus-reward associations: interaction with the ventral striatum. Neuroscience 30:77-86.

Carmichael ST, Price JL (1995a) Limbic connections of the orbital and medial prefrontal cortex in macaque monkeys. J Comp Neurol 363:615-641.

Carmichael ST, Price JL (1995b) Sensory and premotor connections of the orbital and medial prefrontal cortex of macaque monkeys. J Comp Neurol 363:642-664.

Dias R, Robbins TW, Roberts AC (2000) Dissociable forms of inhibitory control within prefrontal cortex with an analog of the Wisconsin card sort test: restriction to novel situations and independence from "on-line" processing. J Neurosci 17:9285-9297.

Dickinson A, Smith J, Mirenowicz J (2000) Dissociation of Pavlovian and 
instrumental incentive learning under dopamine antagonists. Behav Neurosci 114:468-483.

Dolan RJ (1999) On the neurology of morals. Nat Neurosci 2:927-929.

Fantino E (1977) Conditioned reinforcement: choice and information. In: Handbook of operant behavior (Honig WK, Staddon JER, eds), pp 313339. Englewood Cliffs, NJ: Prentice-Hall.

Gallagher M, McMahan RW, Schoenbaum G (1999) Orbitofrontal cortex and representation of incentive value in associative learning. J Neurosci 19:6610-6614.

Ghashghaei HT, Barbas H (2002) Pathways for emotion: interactions of prefrontal and anterior temporal pathways in the amygdala of the rhesus monkey. Neuroscience 115:1261-1279.

Hatfield T, Han JS, Conley M, Gallagher M, Holland P (1996) Neurotoxic lesions of basolateral, but not central, amygdala interfere with pavlovian second-order conditioning and reinforcer devaluation effects. J Neurosci 16:5256-5265.

Holland PC, Gallagher M (2003) Double dissociation of the effects of lesions of basolateral and central amygdala on CS-potentiated feeding and pavlovian-instrumental transfer. Eur J Neurosci 17:1680-1694.

Holland PC, Gallagher M (2004) Amygdala-frontal interactions and reward expectancy. Curr Opin Neurobiol 14:148-155.

Holland PC, Petrovich GD, Gallagher M (2002) The effects of amygdala lesions on conditioned stimulus-potentiated eating in rats. Physiol Behav 76:117-129.

Izquierdo A, Suda RK, Murray EA (2004) Bilateral orbital prefrontal cortex lesions in rhesus monkeys disrupt choices guided by both reward value and reward contingency. J Neurosci 24:7540-7548.

Kita H, Kitae ST (1990) Amygdaloid projections to the frontal cortex and the striatum in the rat. J Comp Neurol 298:40-49.

Kosar E, Grill HJ, Norgren R (1986a) Gustatory cortex in the rat. I. Physiological properties and cytoarchitecture. Brain Res 379:329-341.

Kosar E, Grill HJ, Norgren R (1986b) Gustatory cortex in the rat. II. Thalamocortical projections. Brain Res 379:342-352.

Krettek JE, Price JL (1977) Projections from the amygdaloid complex to the cerebral cortex and thalamus in the rat and cat. J Comp Neurol 172:687-722.

Krushel LA, Van der Kooy D (1988) Visceral cortex: integration of the mucosal sense with limbic information in the rat agranular insular cortex. J Comp Neurol 270:39-54.

Montague PR, Berns GS (2002) Neural economics and the biological substrates of valuation. Neuron 36:265-284.

Parkinson JA, Crofts HS, McGuigan M, Tomic DL, Everitt BJ, Roberts AC
(2001) The role of the primate amygdala in conditioned reinforcement. J Neurosci 21:7770-7780.

Pears A, Parkinson JA, Hopewell L, Everitt BJ, Roberts AC (2003) Lesions of the orbitofrontal but not medial prefrontal cortex disrupt conditioned reinforcement in primates. J Neurosci 23:11189-11201.

Petrovich GD, Setlow B, Holland PC, Gallagher M (2002) Amygdalohypothalamic circuit allows learned cues to override satiety and promote eating. J Neurosci 22:8748-8753.

Pickens CL, Saddoris MP, Setlow B, Gallagher M, Holland PC, Schoenbaum G (2003) Different roles for orbitofrontal cortex and basolateral amygdala in a reinforcer devaluation task. J Neurosci 23:11078-11084.

Pickens CL, Saddoris MP, Gallagher M, Holland PC (2005) Orbitofrontal lesions impair use of cue-outcome associations in a devaluation task. Behav Neurosci 119:317-322.

Rescorla RA (1980) Pavlovian second-order conditioning: studies in associative learning. Hillsdale, NJ: Erlbaum.

Rescorla RA (1988) Pavlovian conditioning: it's not what you think it is. Am Psychol 43:151-160.

Rolls ET (2000) The orbitofrontal cortex and reward. Cereb Cortex 10:284-294.

Saper CB (1982) Convergence of autonomic and limbic connections in the insular cortex of the rat. J Comp Neurol 210:163-173.

Schoenbaum G, Chiba AA, Gallagher M (1998) Orbitofrontal cortex and basolateral amygdala encode expected outcomes during learning. Nat Neurosci 1:155-159.

Schoenbaum G, Chiba AA, Gallagher M (1999) Neural encoding in orbitofrontal cortex and basolateral amygdala during olfactory discrimination learning. J Neurosci 19:1876-1884.

Schoenbaum G, Setlow B, Saddoris MP, Gallagher M (2003) Encoding predicted outcome and acquired value in orbitofrontal cortex during cue sampling depends upon input from basolateral amygdala. Neuron 39:855-867.

Shi CJ, Cassell MD (1998) Cortical, thalamic, and amygdaloid connections of the anterior and posterior insular cortices. J Comp Neurol 399:440-468.

Trapold MA, Overmier JB (1972) The second learning process in instrumental training. In: Classical conditioning II (Black A, Prokasy WF, eds), pp 427-452. New York: Appleton-Century-Crofts.

Tremblay L, Schultz W (1999) Relative reward preference in primate orbitofrontal cortex. Nature 398:704-708.

Weingarten HP (1983) Conditioned cues elicit feeding in sated rats: a role for learning in meal initiation. Science 220:431-433. 